\title{
POLYARTERITIS IN CHILDHOOD
}

\author{
BY \\ A. V. NEALE, M.D., F.R.C.P. \\ (From the Department of Child Health, University of Bristol)
}

With increased study of the mesenchymal tissues and their special reactions to toxic, infective, and allergic factors, the particular pathological grouping, in regard to the place in which the blood vessels are directly involved, has been widely considered. A considerable literature dealing with the pathology and the clinical manifestations of polyarteritis has shown clearly that the connective tissue elements in the vessel walls are, under certain conditions, highly susceptible to inflammatory and necrotizing agents. It must be admitted that the morbid anatomy has been clearly defined, and that certain clinical and experimental observations have lead to some support for an etiological basis of allergy. Nevertheless the precise relationships cannot be considered to be fully understood. In fact it is quite evident that in this field of pathology there is still ample scope for classification, and particularly so in as much as a similar tissue response mechanism is concerned in a wide variety of clinical forms of disease. Polyarteritis may in some cases exist as the only apparent tissue manifestation, but in others there is a more widespread mesenchymal tissue reaction, and an infinity of graduations may occur.

Teilum (1946), in discussing lupus erythematosus disseminatus and related diseases, notes the peculiar fibrinoid necrotizing processes in free connective tissue and in the walls of blood vessels together with miliary epithelioid cell granulomata in the serosa; he proceeds to consider ' a state of allergy induced by specific processes of immunity ' as a basis for the tissue changes. In contrast to lupus erythematosus disseminatus Teilum considers rheumatic fever to be etiologically and clinically a type of specific lesion. However, this author is mindful of the close pathological relationship of lupus erythematosus disseminatus, polyarteritis and arteriolotis granulomatosa (allergica), and inclines to the view that there is a group of ' para-rheumatic' diseases of similar pathogenesis and different etiology, but possessing in common a state of hypersensitivity to bacterial toxins and allergens.

The clinical and experimental observation of Rich (1942) has elucidated possible etiological factors involved in polyarteritis nodosa. There is in these experiments ample evidence for Rich's confident belief that arterial lesions of this type are manifestations of anaphylactic hypersensitivity. Undoubtedly there are widely different types of sensitizing antigens which have the potentiality of causing polyarteritis in man. Galán (1945), after experimental evidence, is of the same opinion. There is, however, the necessary adjuvant factor of constitutional and special tissue susceptibility which tends to defy clinical recognition.

Linkages in pathology are revealed in the cases of rheumatic carditis associated with characteristic polyarteritis nodosa (Neale and Whitfield, 1934). Friedberg and Gross (1934) also describe four cases of periarteritis nodosa in rheumatic fever in which Aschoff bodies were demonstrable in the myocardium. Buckley (1946) points out that in the Libman-Sacks syndrome vascular changes identical with those seen in polyarteritis nodosa are sometimes present, and he records an example in which generalized vasculitis, in association with ulcerous dermal changes, were present; he considered the whole pathology to be a sequel to chronic focal infection.

The experimental work of Selye (1946) is remarkable in so far as it reveals, in certain animals, an arterial reaction with nodular inflammatory changes following injections of adrenal cortical steroid hormone or anterior pituitary extract; this effect is intensified if the animal is receiving excessive amounts of salt. Selye advances the view that in some susceptible persons an overdosage of endogenous cortical hormone, such as may occur in reaction to a sore throat, a nervous shock, or an exposure to cold, etc., may determine the occurrence of tissue responses indistinguishable from that of rheumatic fever and periarteritis nodosa.

It is significant that eosinophilia is only exceptionally present in childhood cases of polyarteritis nodosa, and this bears no inherent relationship to the degree, extent, distribution, or the severity of the lesions. In Wilmer's (1945) two very severe infant cases there was no increase in eosinophils. In a series of twenty-eight cases of polyarteritis in children, two only also had asthma, whereas of three hundred at all ages with the same arterial disease, fifty-four had asthma and 94 per cent. of these revealed hyper-eosinophilia (Wilson, 1945). In the absence of associated asthma, polyarteritis is significantly dissociated from eosinophilic response.

Although the researches of Rich and others have placed a definite allergic responsibility in polyarteritis, clinical observations are by no means so clear, possibly owing to intrinsic difficulties in 
assessing prodromal phenomena in the disease or to the virtual impossibility of exploring sufficiently, at a clinical level, allergic mechanisms of this order. It is of some interest that polyarteritis in childhood is rarely associated with any known common form or type of allergy. This suggests that in polyarteritis a specially individualized arterial receptor mechanism exists for allergen action. By and large, the incidence of this unusual vascular susceptibility, at any age group, appears to be considerably less than the clinical allergies of other tissue systems.

From the clinical aspect no age group appears to be immune in regard to arterial susceptibility and the condition is probably not of great rarity in infancy. The bizarre nature of the clinical manifestations may well be confusing, but Wilmer (1945) has described two cases of polyarteritis in the first month of life. In each of these two infants sepsis was present and the nature of the violent and fulminating course of the disease would indicate a probable peculiarly inherent arterial tissue sensitivity. The intensity of this susceptibility is revealed in the case of a baby aged nine months who died suddenly two months after an attack of acute tonsillitis: the coronary arteries were severely affected with nodular arteritis (Diaz-Rivera and Miller, 1946).

Extended search will probably reveal that polyarteritis is more frequently present than is usually considered. Moreover, there is a strong tendency to some special sectional distribution of the arterial inflammatory reaction in different patients or the effects are dominantly represented in a particular visceral, neural, or cutaneous zone. The prognosis frequently depends upon the more particularly individualized locality of the disease and, in fact, clinical studies suggest that focal arterial reactions, e.g. cerebral, renal, coronary, may be more devastating than quite extensive cutaneous involvement. This tendency to a local dominance is well illustrated by Malamud (1945), who describes a child aged five years in whom polyarteritis selectively involved the meningeal vessels with resultant cerebral damage and decerebrate rigidity. Ophthalmoscopy may be specially helpful; inspection of the choroidal arteries may show fusiform aneurismal dilatations (Goldsmith, 1946) or degrees of retinal detachment (Sampson, 1945).

Although visceral involvement is rightly regarded as a constant menace in polyarteritis, and Bradley (1947) considers the outstanding and special danger to the renal vascular mechanism, cutaneous lesions may exist as the only apparent pathological distribution. Downing (1947) believes that the cutaneous arteries are associately affected in $\mathbf{4 0}$ per cent. of all cases, but it has no special dominance in any age group.

On clinical and pathological evidence the disease may have an exclusively subcutaneous localization. Miescher (1946) described four examples of this kind, and in one form the leucocytoclastic vasculitis of the subpapillary vessels is likened to the papulo- haemorrhagic exanthema of the type peliosis rheumatica. In another, the appearances were similar to that of a papulonecrotic tuberculide, and yet in the others there were variable histological appearances, sometimes approaching granulomatous changes.

The cutaneous type of polyarteritis nodosa may reveal severely acute and alarming effects, more especially owing to the possibility of ischaemic necrosis. Veran (1945) observed massive gangrene of the foot as a result of the disease in a baby aged fifteen months, but in whom general recovery occurred after several months' illness. Particularly mutilating effects were observed by Galán in a boy who developed gross necrosis of the abdominal wall and gangrene of the terminal phalanges of the fingers. In this case there was also a livedo reticularis' and a phasic variation of 0-10 per cent. in the eosinophil polynuclear leucocytes. Despite the severe cutaneous effects, slow but satisfactory recovery occurred. Peripheral panarteritis following scarlet fever is recorded by Hoyne and Smoller (1941).

An appraisal of the clinical signs of the disease in childhood (44 cases) revealed purpuric rash in 34 per cent., other rashes, including urticarial type, in 27 per cent., and palpable nodules in 4.5 per cent. (Keith and Baggenstoss, 1941).

The cutaneous form of polyarteritis offers special advantages in the clinical study of the disease.

\section{Case Reports}

Case 1. D.J.W. was a girl aged nine years, the first child of two. There was no family history of disease. The child's past history was one of pertussis and measles only. In May, 1943, the present illness began. The child developed a fairly severe acute streptococcal tonsillitis with moderate submaxillary adenitis. A few days later ' rheumatic' pains commenced in the shoulders, back and legs, and shortly afterwards an 'unusual ' skin rash made its appearance. The child felt generally ill and remained in bed until admitted to hospital. Under hospital observation it was clear that a somewhat unusual syndrome was present. She was irritable and in continued discomfort and had variable limb pains. The two outstanding visible signs were the persistent flexural position of the limbs in bed and a very marked mottled rash all over the body, especially marked on the limbs. The rash was similar to that of 'cadaveric staining', and was striking in so far as the face was also affected and the intensity varied from day to day, sometimes being peculiarly prominent and unlike any other known rash. There was a fluctuating appearance and disappearance of subcutaneous tender nodules which left no doubt that the subcutaneous arteries were affected by acute focal, nodular, and inflammatory changes. There was never, in this girl, any evidence of tissue or skin necrosis, which presumably indicated that the arterial lumen was in no area 
Fig. 3.-D.J.W. later. No signs of disease, but 'meshwork' staining of lower limbs still present.

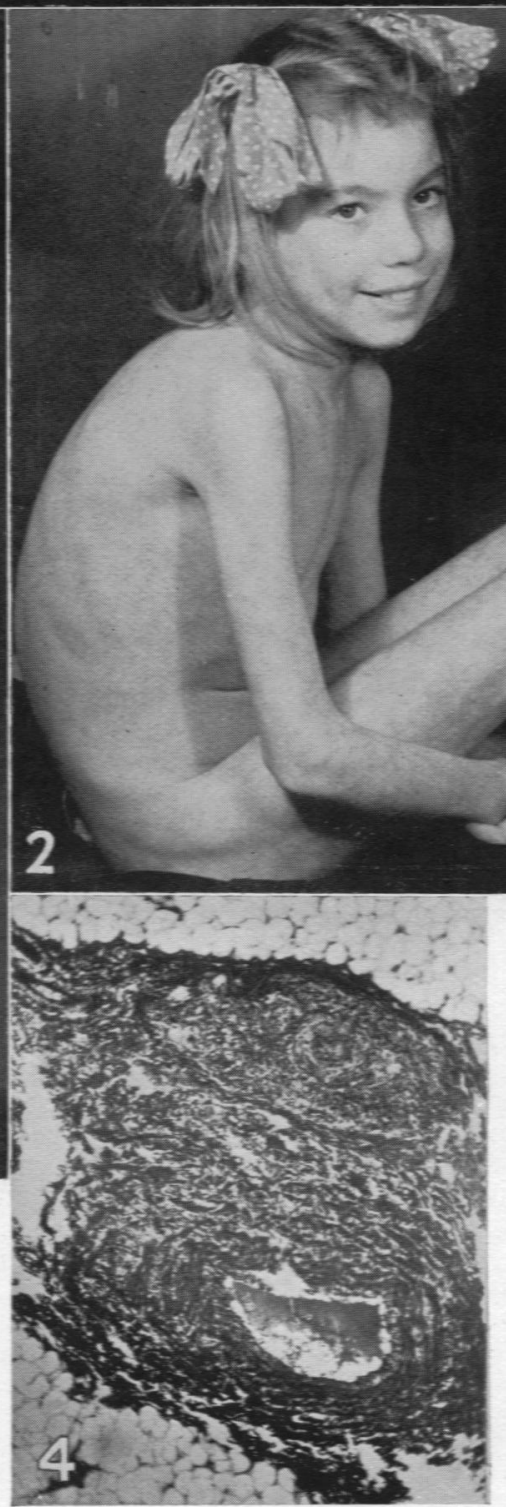

FiG. 1.-D.J.W. A few weeks after the onset of acute polyarteritis. Note the flexion attitude and the mottled rash on the limbs.

FIG. 2.-D.J.W. in a phase of clinical improvement. Rash and nodules visible.

FIG. 4.-D.J.W. BIOPSY OF SKIN.-Proliferation of subendothelioid connective tissue of the intima; oedema and necrobiotic changes in the media with disarrangement of the muscle fibres, cellular infiltration, and fibrosis of the adventitia. Occasional necrosis of perivascular tissue with invasion of granulation tissue with foreign body giant cells. Elastica was intact in some arteries and disrupted in others. 


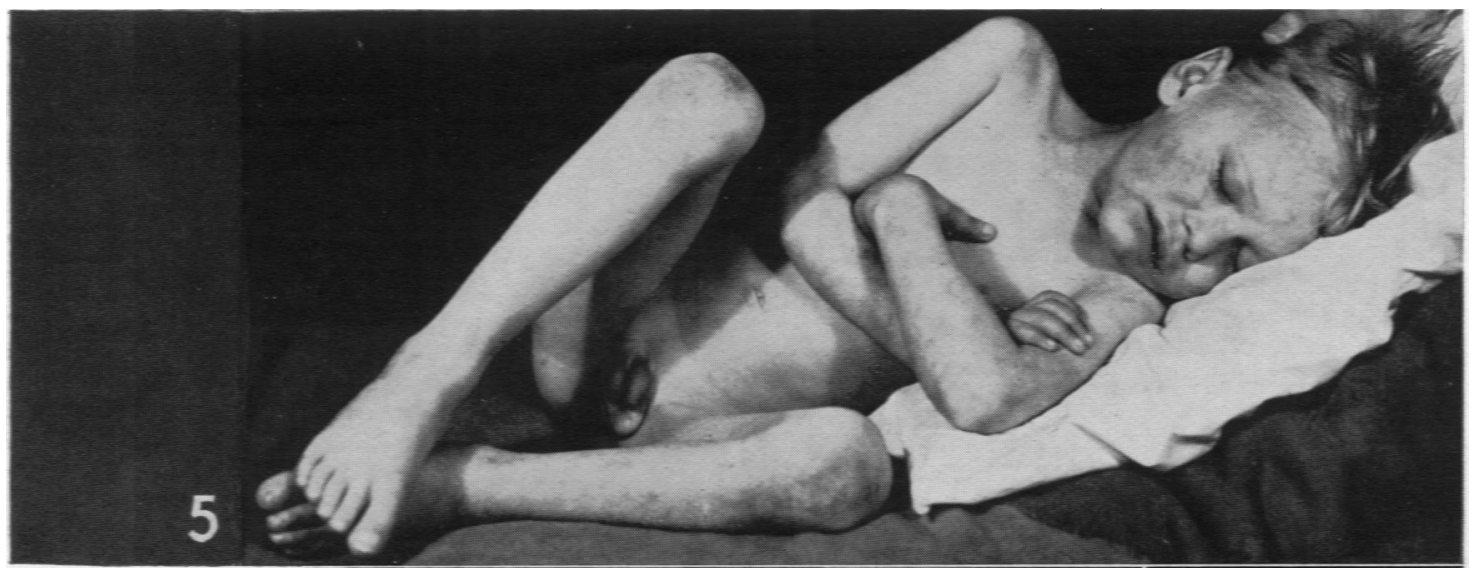

FIG. 5.-H.J. The early acute phase. Note flexion posture; cutaneous nodules, and characteristic rash on the limbs and face.

FIG. 6.-H.J. A phase of great clinical improvement. Note residual appearance of limbs and slight oedema of the feet.

FIG. 7.-H.J. Dermal necrosis in final acute phase. Note nearby arterial nodules.

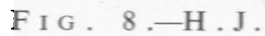
I s ch a e $\mathrm{m} \mathrm{ic}$ effects of fingers in final acute phase of necrotizing obliterative arteritis.

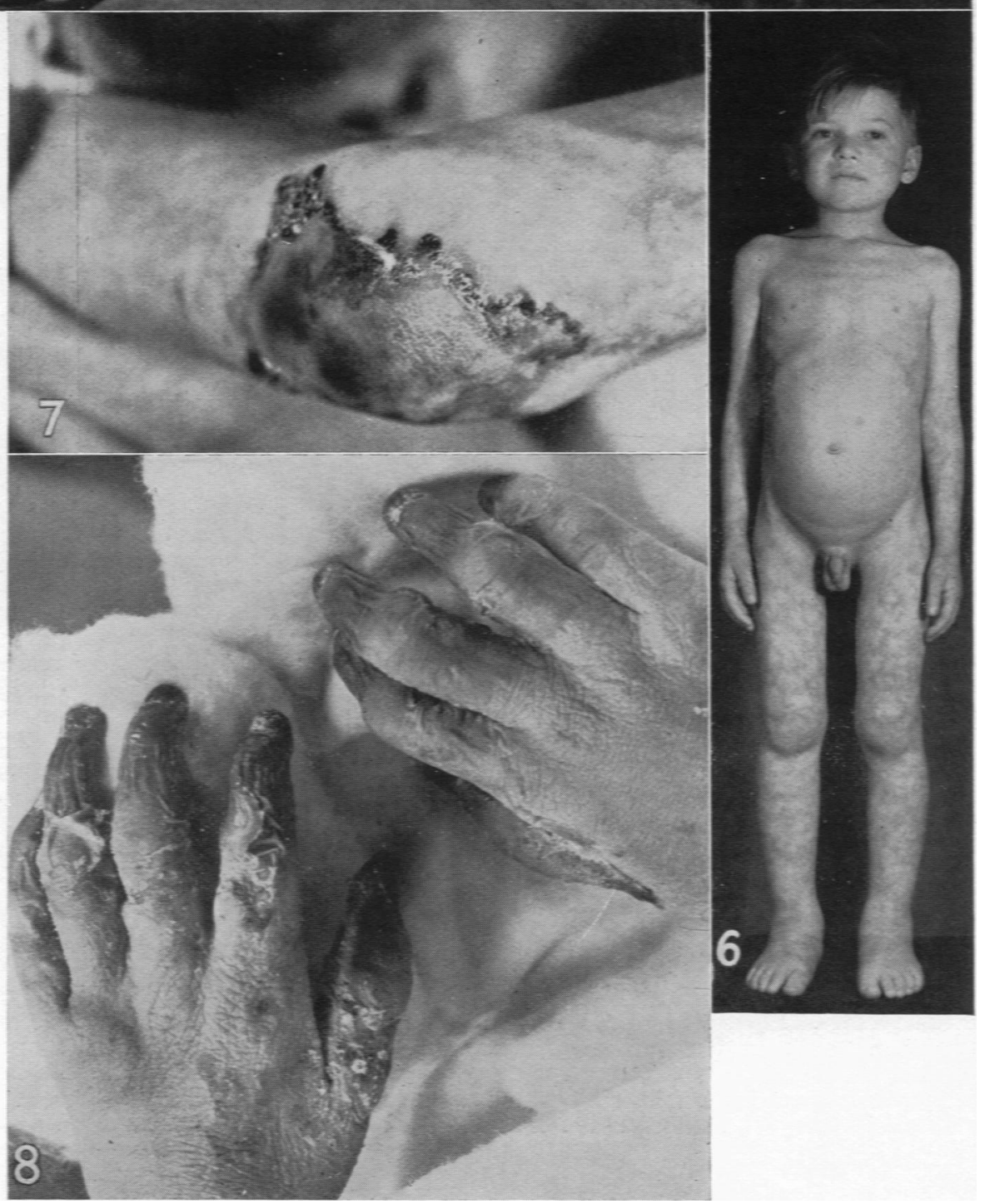


obliterated. There was no evidence at any time of any arterial disease in the deeper structures or in the viscera. Repeated examination revealed no evidence of renal involvement and the heart remained clinically and electrocardiographically normal. The outstanding clinical feature was the persistent rash, traces of which can still be seen (three years later). Considerable general wasting accompanied the illness, and over an observed course of eight months (May, 1943, to February, 1944) there was an undulating pyrexia with maximal levels around $103^{\circ}$. The pulse rarely reached above 120 and the respiratory rate was 30 . There was no abnormal sweating. There was a persistent leucocytosis of 15,000 per c.mm., but eosinophils were never more than 4 per cent. Blood cultures were sterile.

A biopsy of the skin (forearm) was done on March 14, 1944. The majority of the arteries in the subcutaneous tissue, and in a lesser degree those of the cutis vera, showed severe pathological changes. There was proliferation of the subendothelial connective tissues of the intima, necrobiotic changes of the media, and proliferation of the vascular granulation tissues of the media with much disarrangement of the muscle fibres. The cellular infiltrations consisted of neutrophil polymorphs, lymphocytes, monocytes, and a few eosinophils. The infiltration extended sometimes throughout the whole vessel wall and often diffusely into the perivascular connective tissue. Foci of extensive necrosis were seen in the perivascular tissue, and a form of giant cell granulation tissue could be seen in some areas. There was occasional disruption of the muscle fibres. The picture was that of panarteritis nodosa.

From time to time the child had severe limb pains and very strongly resented being handled or moved. Skin nodules appeared and disappeared, and the whole illness presented considerable phasic variability. Many symptomatic remedies were prescribed, but it seemed clear that the disease was refractory. In view of the possibility of retained tonsil sepsis, tonsillectomy was performed, but no appreciable improvement occurred. Three months later a very severe exacerbation of all the symptoms and signs appeared with some oedema of the feet. The mesh-like general rash and erythematous nodular foci again became prominent. The clinical progress indicated a waxing and waning of arteritis nodosa of cutaneous form.

After a prolonged stay in hospital, gradual improvement occurred, although limb pains occurred when she was unduly warm in bed. The rash gradually diminished.

In the following two years occasional crops of periarterial nodules made their appearance and were usually accompanied by limb pains and some days of immobility. Finally, in 1945, she seemed to be free from all effects of the prolonged illness. One year later she developed diphtheria and was given adequate doses of anti-serum without any adverse effects. In 1948, at the age of thirteen years, she was clinically well. Puberty occurred normally and menstruation appeared.

Case 2. H.J. was a boy aged eight years and the fifth child of seven. There was no family history of disease, and the child had a past history of measles only.

This boy was admitted to hospital in June, 1943, seventeen days after a sore throat, and actually during the same time as the previous case. The clinical picture at that time was identical. Thirteen days before admission he had received sulphonamide and three days later a macular rash appeared all over the body and also numerous red-purple focal swellings. Pains in the joints, varying in the manner of rheumatic fever, also occurred. There was no evidence of any visceral disturbance.

Histology of a subcutaneous nodule confirmed the diagnosis of periarteritis nodosa. The blood showed a fluctuating neutrophil leucocytosis, reaching a maximum of 21,400 , but at no time did the eosinophils exceed 1.5 per cent.

This boy was particularly troubled with limb pains, and on several occasions temporary joint swellings with small effusions appeared. Clinically the intensity of the disease was more severe in this case. The 'mesh-work' erythema was very marked and quite prominent on the face. The feet were swollen and very painful and there were many subcutaneous nodules along the course of the arteries. The heart was clinically normal and electrocardiograms were normal. A variable pyrexia with maxima of $103^{\circ}$ continued over many weeks. Intensive doses of penicillin had no favourable effect, and sulphathiazole was given without any response. Over a period of one year the disease varied in intensity, but eventually he became ambulatory and comfortable, and his mother thought he was very well. However, a few cutaneous arterial nodules continued to appear at intervals and the 'cadaveric' staining on the limbs was persistent. Eighteen months after the onset the boy was gaining weight and had no symptoms, but he had clearly not reached a phase of real health. Three weeks later he had a 'cold' and a recurrence of the previous limb pains and a marked accentuation of the cutaneous erythema. His condition quickly deteriorated and necrosis appeared in the fingers and in some other skin areas. Necropsy revealed no evidence of any visceral involvement in the arterial disease. There was no tonsil sepsis or any other evidence of active focal infection. There was no renal pathology.

\section{Discussion}

These two cases of polyarteritis indicate (1) that the disease reaction may be apparently confined to a particular section of the arterial system; (2) that such pathological response may have a prolonged and clinically fluctuant course and be compatible with a satisfactory recovery without sequelae; or, 
(3) by an acute exacerbation, lead to quickly spreading ischaemic necrotic changes.

The clinical features of the dermal form of arteritis are distinguishable more particularly by the occurrence of painful nodular swellings visible and palpable in the skin. The arterial nodules may be extremely numerous and yet apparently the incidence of intra-arterial thrombosis is minimal. This is particularly indicated in the case of D.J.W., in whom it was not possible at any time during her relapsing illness of many months to detect any effects arising out of lumenal occlusion. The type of generalized rash is peculiarly characteristic in its meshwork of bluish discoloration and resemblance to that seen in cadaveric staining; this is most marked on the lower limbs and is very persistent throughout, and for some time after the active phase of the disease has subsided. In the two cases here recorded the face showed a variable general swelling in relationship to the periodic intensification of the rash. The mucous membranes were unaffected but conjunctival suffusion was present.

No definite etiological factors were discovered, but in each case the arteritis was preceded by rather severe sore throat, so that bacterial toxaemia was probably causally related. In neither case could any definable allergic susceptibility be established in an individual or familial basis. The bouts of fever may be merely related to pyrogenic products in the necrotizing arteritis.

\section{Treatment}

The life history of the disease and its refractory nature to any known specific therapeutic measure reduces the treatment to symptomatic remedies for pain, insomnia, etc. The tendency for lower limb contractures, owing to prolonged decubitus immobilization, must be overcome by suitable temporary splinting. The peripheral vascular ischaemic effects might be in part remedied by nicotinic acid. Benadryl and other anti-histamine drugs have also received clinical trial, without, however, any detectable effect.

\section{Summary}

Some considerations of the possible etiological relationships in polyarteritis in childhood are presented and two cases of the 'dermal' form are recorded. A prolonged illness, with an ever-present menace of possible visceral involvement, was successfully negotiated and clinical recovery without sequelae occurred in one child. In another child the prolonged and variable clinical course seemed to have reached a favourable outcome, but unexpectedly a sudden and intense relapse quickly progressed to acute obliterative cutaneous arteritis and spreading ischaemic necrosis.

\section{REFERENCES}

Bogeart, L. van et al., (1932). Ann. Med., 31, 530. Bradley, E. J. (1947). J. Pediat., 31, 78.

Buckley, S. (1946). Helvet. Paediat. Acta., 1, 524.

Downing, J. G. (1947). New Eng. J. Med., 237, 906.

Diaz-Rivera, N. S., and Miller, A. J. (1946). Annals Int. Med., 24, 420.

Friedberg, C. K., and Gross, L. (1934). Arch. Int. Med., $54,170$.

Galán, E. (1945). Bol. Soc. Cubana Pediat., 17, 293.

Goldsmith, J. (1946). Amer. J. Ophthal., 29, 435.

Hoyne, A. L., and Smollar, L. (1941). J. Pediat., 18, 242.

Keith, H. M., and Baggenstoss, A. H. (1941). J. Pediat., 18, 494.

Malamud, N. (1945). J. Neuropath. exp. Neurol., 4, 88.

Middleton, W. S., and McCarter, J. C. (1935). J. Amer. Med. Sc., 190, 291.

Miescher, G. (1946). Dermatologica Basel, 92, 225.

Neale, A. V., and Whitfield, G. W. (1934). Brit. med. J., 2, 104

Rich, A. R. (1942). Bull. Johns Hopk. Hosp., 71, 123.

- , and Gregor, J. E. (1943). Ibid., 72, 65. (1945). Ibid., $77,43$.

Sampson, R. (1945). Brit. J. Ophthal., 29, 282.

Selye, H. (1946). J. clin. Endocrinal, 6, 117.

Teilum, G. (1946). Acta. med. scand., 123, 126.

Wilmer, H. A. (1945). Bull. Johns Hopk. Hosp., 77, 275.

Wilson, K. S., and Alexander, H. L. (1945). J. Lab. clin. Med., 30, 195.

Veran, P. (1945). Arch. Mal. Coeur., 38, 149. 